\title{
Fighting an Infodemic in the Time of the COVID-19 Pandemic in India: Leveraging Technology and Social Media
}

\author{
Sakshi Jindal, Gaurav Anand \\ Tata Consultancy Services, Medical Writing, Noida, India
}

\section{Dear Editor,}

We read with interest the article titled "Impact of rumors and misinformation on COVID-19 in social media" published in your esteemed journal [1]. As a continuation of the points made in that article, in this letter, we would like to discuss how technology and social media are being or should be leveraged to fight the coronavirus disease 2019 (COVID-19) "infodemic" and COVID-19-related misinformation in India. These strategies can further be replicated in the rest of the world.

Misinformation is spreading through the use of technology (smartphones, Internet/mobile applications [e.g., WhatsApp]), social media platforms (e.g., Facebook, Twitter, YouTube, TikTok), and traditional mass media, and thus needs better regulation. This situation is exacerbated by the particularly low levels of health literacy in India [2], and it may not be possible for the majority of people in India to identify correct information from the vast ocean of available media, in which there are massive amounts of misinformation.

To fight this infodemic - or, in other words, this menace of misinformation - the government of India is promoting the use of official websites such as the Ministry of Health and Fam-

Corresponding author: Sakshi Jindal, MSc

Tata Consultancy Services, Medical Writing, Noida 201309, India

E-mail: sakshi.pharma3@gmail.com

This is an Open Access article distributed under the terms of the Creative Commons Attribution Non-Commercial License (https://creativecommons.org/licenses/by$\mathrm{nc} / 4.0 /$ ) which permits unrestricted non-commercial use, distribution, and reproduction in any medium, provided the original work is properly cited. ily Welfare (MoHFW) [3] when seeking information on COVID-19 or for access to real-time disease data. Searches pertaining to "coronavirus," "COVID-19," or related terms on social media platforms are directed to a reliable source like the MoHFW website [3] or an approved government website, as seen on Google, YouTube, and Twitter. A 24-hour free hotline has been set up in India, where healthcare experts/health authorities respond to questions/concerns raised by public.

In the fight against COVID-19, government is promoting the use of a mobile health app, the "Aarogya Setu" app, which has currently been downloaded by approximately 157 million people in India. This app provides correct and updated information on COVID-19 cases within a $0.5 \mathrm{~km}$ to $10 \mathrm{~km}$ radius of the user and facilitates contact tracing of infected people, among other benefits. The app would be increasingly instrumental in fighting the infodemic/misinformation if it could be modified to have a fake news management component, where false claims about Ayurvedic, homeopathic, and immunity-booster medicines or religious/spiritual approaches to COVID-19 disease management can be debunked by competent authorities.

The manual fact checking or filtering of keywords cannot control the content of the infodemic shared on the Internet, as seen in an Oxford study [4], wherein, based on an analysis of a sample of 225 pieces of misinformation from January 2020 to March 2020, it was found that the majority (88\%) of the sample rated as false or misleading by independent fact-checkers appeared on social media. We propose to use a combination 
of rule-based engine (non-cognitive text analytics) and an artificial intelligence application based on cognitive machine learning (ML) which can explore, analyze, and filter the information effectively. The ML-based application can apply cognitive text, image, and/or video analytics to data; evaluate the content with the approved data; and filter it for approval (stringent criteria of at least a $90 \%$ match) or rejection for upload.

Additionally, health agencies can use Google Trends to curb misinformation. Further, response to COVID-19 in India can be enhanced by developing comprehensive solutions for frontline healthcare workers and rapid response teams using a mHealth app [5]. The features of the mHealth app could include sharing of clinical notes and treatment algorithms drafted by experts and modified periodically in light of available real-time information, best practices shared globally and adapted to fit the Indian healthcare landscape, and a chat box for Q\&A led by competent health professionals.

Therefore, we conclude that the infodemic can be fought using the same technology that is facilitating misinformation, thereby making us better equipped to face such challenges in the future in the wake of outbreaks of infectious diseases such as COVID-19.

\section{CONFLICT OF INTEREST}

The authors have no conflicts of interest associated with the material presented in this paper.

\section{ORCID}

Sakshi Jindal https://orcid.org/0000-0002-6368-5794

Gaurav Anand https://orcid.org/0000-0001-9227-7143

\section{REFERENCES}

1. Tasnim S, Hossain MM, Mazumder H. Impact of rumors and misinformation on COVID-19 in social media. J Prev Med Public Health 2020;53(3):171-174.

2. Kumar R. Health literacy a must to empower patients. Tribune 2019 Apr 4 [cited 2020 Jul 20]. Available from: https://www.tribuneindia.com/news/archive/comment/health-literacy-amust-to-empower-patients-752945.

3. Ministry of Health and Family Welfare, Government of India. COVID-19 India [cited 2020 Sep 16]. Available from: https:// www.mohfw.gov.in/.

4. Philip Howard P, Nielson RK, Newman N, Brennan JS. The COVID-19 'infodemic': what does the misinformation landscape look like and how can we respond? [cited 2020 Sep 7]. Available from: https://www.oii.ox.ac.uk/blog/the-covid-19-infodemic-what-does-the-misinformation-landscape-look-likeand-how-can-we-respond/.

5. Bassi A, Arfin S, John O, Jha V. An overview of mobile applications (apps) to support the coronavirus disease 2019 response in India. Indian J Med Res 2020;151(5):468-473. 